Allergology today

\title{
Tolerierter Insektenstich verbessert Lebensqualität nach SIT
}

\begin{abstract}
Welche Auswirkungen eine spezifische Immuntherapie (SIT) bei Insektengiftallergikern auf die Lebensqualität und die Angst vor Insektenstichen hat, wurde bislang wenig untersucht. Eine Arbeitsgruppe um Jan Adelmeyer von der Universitätsklinik für Dermatologie und Allergologie in Marburg befragte dazu 61 Insektengiftallergiker, bei denen die SIT durchschnittlich neun Jahre zurücklag. 31 von ihnen waren zwischenzeitlich erneut gestochen worden. Obwohl fast alle Patienten von der Wirksamkeit der SIT überzeugt waren, versuchten die meisten, einen Stich zu vermeiden. Patienten, die einen Feldstich toleriert hatten und
\end{abstract}

keine Notfallmedikation benötigten, fühlten sich in ihrem Alltagsverhalten am geringsten beeinträchtigt und hatten am seltensten ausgeprägte Angst davor, erneut gestochen zu werden. Eine Stichprovokation nach einer SIT sei somit nicht nur für den Nachweis der Wirksamkeit wesentlich, sondern habe auch günstige Auswirkungen auf die Lebensqualität der Betroffenen, schlussfolgerte Adelmeyer. Angelika Bauer-Delto

Adelmeyer J, Möbs C, Pfützner W. Long-term analysis of quality-of-life in patients allergic to hymenoptera venom who were treated with specific immunotherapy

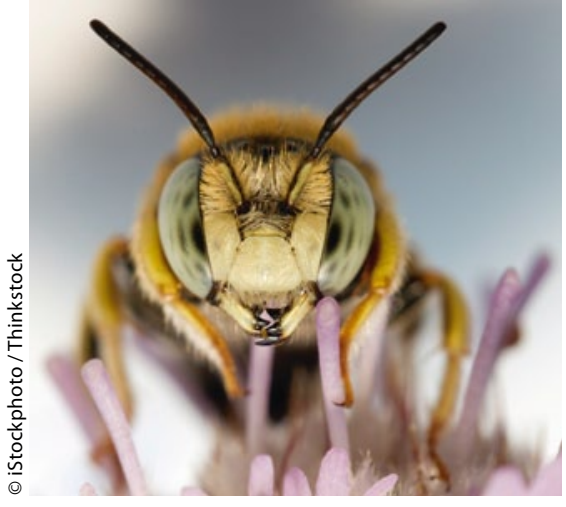

Verbreitet auch nach einer Hyposensibilisierung noch Unbehagen

\section{Gen-Food für Nahrungsmittelallergiker?}

Für Patienten mit Nahrungsmittelallergien könnten hypoallergene Lebensmittel eine Alternative sein. Um Allergene in Pflanzen gentechnisch zu supprimieren, nutzen verschiedene Forschergruppen die Methode der RNA-Interferenz (RNAi). Das Prinzip basiert darauf, DNA-Konstrukte, welche die cDNA des Zielproteins in Sense- und Anti-

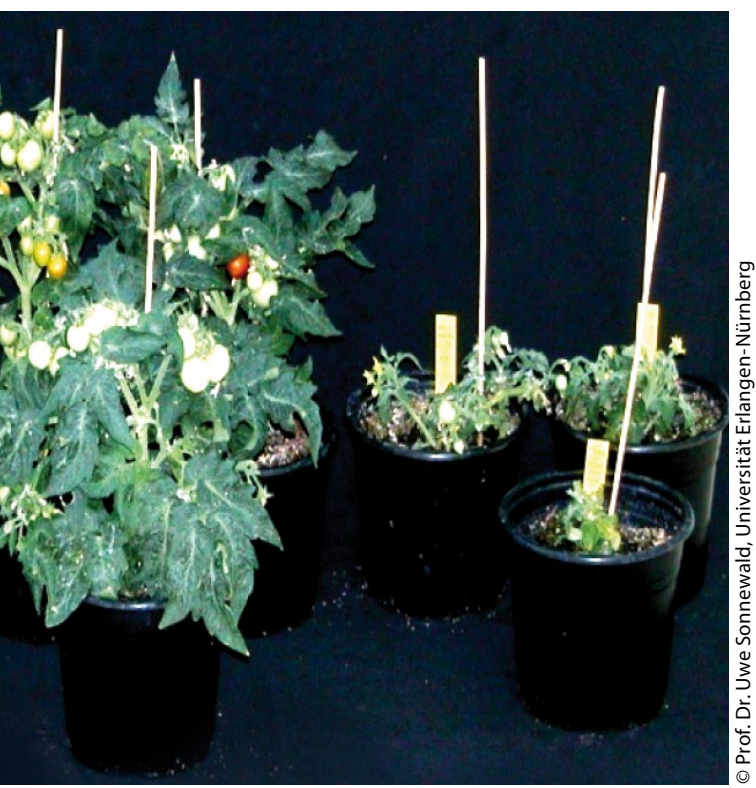

Wildtyp-Tomate (wt), Phänotyp bei Profilin-Supprimierung (Lyce1_RNAi) und nach Rekonstitution mit Hefeprofilin (Lyce1_RNAi/YPFN)
sense-Orientierung enthalten, in Zellen einzuschleusen. Diese werden dann transkribiert und führen als small interfering-RNA (siRNA) durch die Hemmung der Translation zu einem sogenannten "gene silencing", erklärte Dr. Stephan Scheurer vom PaulEhrlich-Institut, Langen.

Mit dieser Technologie gelang es bereits bei zahlreichen Lebensmitteln, Hauptallergene sehr stabil und über mehrere Generationen zu supprimieren. Die transgenen Pflanzen zeigen sowohl in vitro als auch im Hauttest eine deutlich reduzierte allergene Potenz. Eine Erklärung für eine beobachtete Restreaktivität könnte sein, dass es sich bei der RNAi-Methode um eine Suppression und nicht um ein völliges Ausschalten von Allergenen handelt, sagte Scheurer. Zudem ist die Methode sehr spezifisch, sodass möglicherweise nicht alle Isoformen einer Allergenfamilie erfasst werden. Außerdem weisen viele Patienten Polysensibilisierungen auf verschiedene Allergene einer Pflanze auf; neuere Forschungsarbeiten zielen daher darauf ab, mehrere Allergene simultan zu supprimieren.

Die umfangreichsten Studien wurden bislang mit Tomaten durchgeführt. In ersten Untersuchungen wurde das Lipid-TransferProtein Lyc e 3 supprimiert. Dies hatte keinen Einfluss auf den Phänotyp der Pflanzen. In Pricktests bei fünf Tomatenallergikern mit Sensibilisierung auf Lyc e 3 zeigten drei keine und die beiden anderen nur moderate Reak- tionen auf allergensupprimierte Tomaten. Ein "gene silencing" von Lyc e 1 (Profilin) hatte allerdings gravierende Auswirkungen auf das Wachstum der Pflanzen. Durch Substitution mit Profilin aus der Bäckerhefe gelang es, den Phänotyp der transgenen Pflanzen gemäß dem Wildtyp zu rekonstituieren [Le L et al. FASEB 2010]. Diese Methode mache jedoch eine umfassende Risikobewertung der mit dem Profilin substituierten Pflanzen erforderlich, betonte Scheurer. Profilin aus der Bäckerhefe wurde bislang nicht als Allergen beschrieben. Tomatenallergiker mit Reaktivität auf Lyc e 1 wiesen keine Sensibilisierung gegen das Bäckerhefeprofilin auf, bei Orangenallergikern mit Sensibilisierung gegen Profiline wurde allerdings eine Co-Sensibilisierung auf Hefeprofilin nachgewiesen. Eine allergene Potenz für ein bestimmtes Patientenkollektiv kann daher nicht ausgeschlossen werden.

Die Limitation der Methode liege heute vor allem in der geringen Akzeptanz gentechnisch veränderter Lebensmittel in der Öffentlichkeit und bei betroffenen Allergikern, so Scheurer. Umfangreiche Risikobewertungen, die Untersuchung des gesamten Expressionsprofils einer Pflanze, mögliche Auswirkungen einer Allergensupprimierung auf biologische Funktionen und eine klinische Evaluation von hypoallergenem GenFood seien nun die Aufgaben der Zukunft.

Angelika Bauer-Delto

Scheurer S. Genetically engineered hypoallergenic foods: potential and limitations 